\title{
Impermeable Graphenic Encasement of Bacteria
}

\author{
Nihar Mohanty, ${ }^{\dagger}$ Monica Fahrenholtz, $^{\dagger}$ Ashvin Nagaraja, ${ }^{\ddagger}$ Daniel Boyle, ${ }^{\S}$ and Vikas Berry ${ }^{*}+$ \\ ${ }^{\dagger}$ Department of Chemical Engineering, ${ }^{\ddagger}$ Department of Electrical Engineering, and ${ }^{\S}$ Division of Biology, Kansas State University, \\ Manhattan, Kansas 66506
}

Supporting Information

ABSTRACT: Transmission electron microscopy (TEM) of hygroscopic, permeable, and electron-absorbing biological cells has been an important challenge due to the volumetric shrinkage, electrostatic charging, and structural degradation of cells under high vacuum and fixed electron beam. ${ }^{9}$ Here we show that bacterial cells can be encased within a graphenic chamber to preserve their dimensional and topological characteristics under high vacuum $\left(10^{-5}\right.$ Torr $)$ and beam current

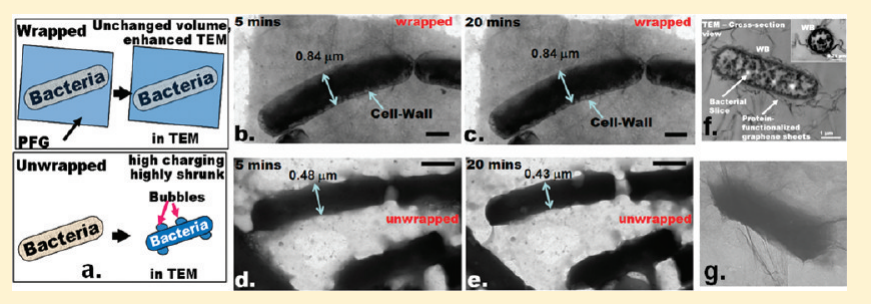
$\left(150 \mathrm{~A} / \mathrm{cm}^{2}\right)$. The strongly repelling $\pi$ clouds in the interstitial sites of graphene's lattice ${ }^{4}$ reduces the graphene-encased-cell's permeability from $7.6-20 \mathrm{~nm} / \mathrm{s}$ to $0 \mathrm{~nm} / \mathrm{s}$. The $\mathrm{C}-\mathrm{C}$ bond flexibility, ${ }^{5,6}$ enables conformal encasement of cells. Additionally, graphene's high Young's modulus ${ }^{6,7}$ retains cell's structural integrity under TEM conditions, while its high electrical ${ }^{8}$ and thermal conductivity ${ }^{9}$ significantly abates electrostatic charging. We envision that the graphenic encasement approach will facilitate real-time TEM imaging of fluidic samples and potentially biochemical activity.

KEYWORDS: Graphene, bacteria, impermeability, nanotechnology, TEM, membrane

Cor the last 80 years, TEM imaging has been a key component 1 in the evolution of science and technology. 1,2 Currently, there are two common routes to conduct room-temperature TEM imaging of wet cells: ${ }^{1-3}$ (1) Environmental TEMs (ETEMs), where cells are imaged at low vacuum $(\sim 1$ Torr $)$, thereby reducing the pressure drop between the intracellular and extracellular regions of the cell; ${ }^{10-16}$ (2) microfluidic TEM where thin, impermeable, and electron transparent chambers are fabricated lithographically and the cells are trapped within it. $^{17-20}$ While, ETEM has shown tremendous promise in native cell imaging, the low vacuum is high enough to shrink the cells. ${ }^{1,2}$ On the other hand, the microfluidic encasement achieves wetphase imaging even with a regular TEM. However, the technique requires extensive lithography, and does not provide a conductive sink to reduce charging. ${ }^{1,20}$ Alternatively, cryogenic solidphase TEM imaging of biological cells has received great attention due to its ease of use; ${ }^{1,2}$ here, the sample is frozen/solidified and therefore retains its volatile content. However, this technique does not allow "liquid-phase" imaging.

A solution to the above-mentioned challenge is to confine the cells within an easy-to-apply impermeable and electron-transparent encasement, which retains the cellular water content while enabling TEM imaging. This work shows that few-atoms-thick graphenic sheets have a unique combination of properties (mentioned above and more), making it an ideal candidate for cellular encasement for TEM imaging. The following properties of graphenic sheets are leveraged: (a) impermeability, ${ }^{5,21}$ the close-packing of carbon atoms in graphene leaves ultrasmall interstitial space with high $\pi$-electron density restricting the passage of even the small atoms, such as helium; (b) high electron transparency, ${ }^{22}$ high-momentum electrons can transmit through monolayer or multilayer $(1-10 \mathrm{~nm})$ graphene, which enables facile TEM imaging; (c) flexibility ${ }^{6,7}$ (which enables graphene to be rolled into carbon nanotubes) allows the sheets to conformally wrap the cells or other macroscale particles; (d) mechanical strength, ${ }^{5,23}$ graphene's high yield strength enables it to sustain the high-pressure differences, similar to that between the intracellular region and external vacuum of a TEM; (e) high electrical conductivity, ${ }^{8,22}$ the mobile $\pi$ electrons of graphene significantly reduce the electrostatic charge buildup under electron microscopy (EM); (f) high thermal conductance, ${ }^{9,22}$ graphene's high phonon conductivity dissipates the heat generated from electron bombardment. Clearly, this unique combination of properties makes graphene an ideal nanomaterial for wet-phase imaging under TEM, while preserving intracellular volatile content of the cell under high vacuum.

This paper demonstrates that protein-functionalized graphene (PFG) can specifically wrap bacteria, thus completely encasing them. The wrapping subsequently facilitates their effective wetphase TEM imaging. This technique is shown for Gram-positive bacteria, Bacillus subtilis. These bacteria have about $70 \%$ water content (volumetric) with a cell wall thickness of $16-30 \mathrm{~nm}^{24}$ and were kept unstained (see Supporting Information). Briefly, an aqueous suspension of graphene oxide (GO) sheets with area between 20 and $600 \mu \mathrm{m}^{2}$ was prepared via the modified Hummer's method. ${ }^{25,26}$ To enhance wrapping, the GO was

Received: December 8, 2010

Revised: January 16, 2011

Published: January 27, 2011 


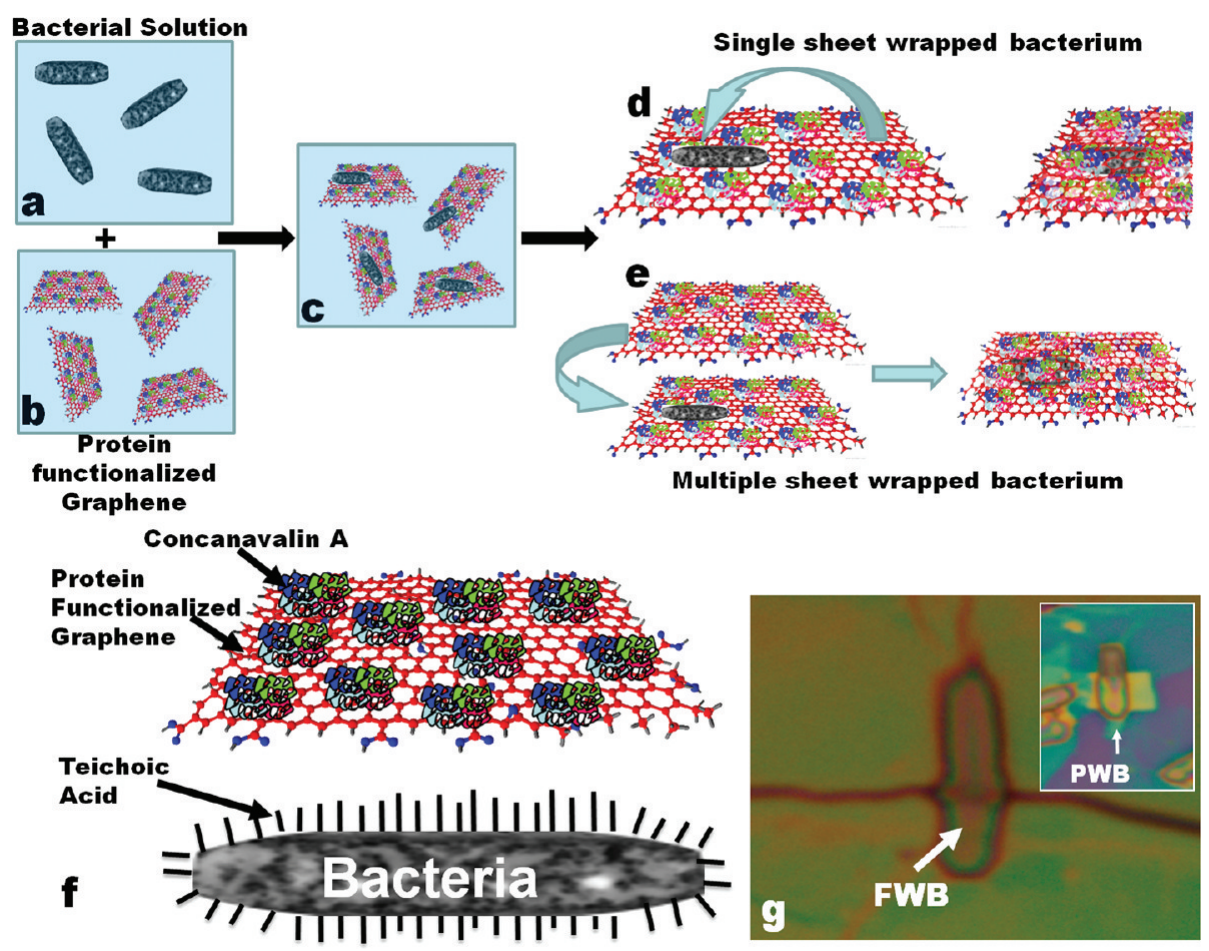

Figure 1. (a-e) Schematic diagram showing the steps involved in the bacterial wrapping by the PFG sheets. (f) A schematic showing the highly specific interaction between the CA on the PFG sheets and the teichoic acid moieties on the bacterial cell wall. ( $\mathrm{g}$ ) An optical microscope image of a fully wrapped bacterium (FWB) and a partially wrapped bacterium (PWB, top right inset).

covalently bonded to the amine groups on membrane-binding protein, ${ }^{25}$ Concanavalin-A (CA), which has specific-affinity to the polyteichoic acid moieties on the bacterial cell wall. ${ }^{27}$ These protein-covered sheets are referred to as PFGs in this paper. To confirm CA functionalization, fluorescein isothiocyanate (FITC) dye labeled CA was used. Substrate-deposited PFGs showed a faint green fluorescence (owing to quenching by $\mathrm{GO}^{28}$ ), confirming the successful functionalization (see Figure S1, Supporting Information). Purified bacterial cell suspension (cultured overnight in nutrient agar media, pelleted down, washed, and diluted to $\sim 10^{3}$ cells $/ \mathrm{mL}$ in deionized (DI) water) was mixed with a fresh PFG suspension $(\sim 10 \mathrm{mg} / \mathrm{mL})$. This led to a visible clouding of the suspension, which we attribute to the bacterial encasement by the PFGs. It is expected that the large area of PFG sheets will strengthen its interfacing with bacteria. The PFG encasement/wrapping mechanism is proposed to be a sequential multipoint attachment process, as favored by minimization of Gibbs energy

$$
\sum_{i=1}^{n}\left(\mu_{\mathrm{G}+{ }_{i}} N_{\mathrm{G}+i}+\mu_{\mathrm{B}-i} N_{\mathrm{B}-i}\right)>\sum_{i=1}^{n}\left(\mu_{\mathrm{GB} \pm i} N_{\mathrm{GB} \pm i}\right)
$$

where $\mu$ is the chemical potential, $N$ is the number of sites, and the subscripts $\mathrm{G}+$ and $\mathrm{B}-$ are the $\mathrm{CA}$ and teichoic acid sites on PFG and bacteria, respectively). ${ }^{29}$ Further, the sizable reduction of free energy originating from the reduction of chemical potential via the highly specific ligand-receptor interaction is expected to offset the ligand-receptor bond stretching during wrapping. ${ }^{29}$ The anticipated wrapping process is depicted in panels a-e of Figure 1. The negative control experiments with $\mathrm{GO}$ in the absence of CA yielded no wrapping, attributed to the increase in free energy with the proximity between negatively charged GO and teichoic acid.
The cross section of the PFG-wrapped bacteria was characterized by cutting $90 \mathrm{~nm}$ thick sections (slices) of wrapped bacteria using a microtome (EM UC7, Leica, Inc.). These sections were analyzed under the TEM (CM100, $100 \mathrm{kV}$, FEI Inc.). $90 \pm 10 \%$ of the bacteria were fully wrapped (Figures S2 and S3, Supporting Information), while the others were partially wrapped. The high wrapping efficiency of the process is attributed to (a) the larger area $\left(\sim 20-600 \mu \mathrm{m}^{2}\right)$ of the PFG sheets, compared to the bacterial surface area $\left(2-6 \mu \mathrm{m}^{2}\right)$ (see supporting videos SV1 and SV2, Supporting Information) and (b) the higher concentration of PFGs in comparison to bacteria. A majority of the cells were wrapped with multilayered $\mathrm{PFG}$ sheets $\sim 2$ to $5 \mathrm{~nm}$ thick (corresponding to $\sim 1$ to $7 \mathrm{GO}$ layers $(\mathrm{CA} \text { is } \sim 1 \mathrm{~nm})^{30}$ ) as shown in Figure 2a (see Figures S1, S2, and S3, Supporting Information). The thicker sheets are expected to exhibit a higher impermeability, while maintaining electron transparency. ${ }^{21,22}$ Further, the wrapped sheets conformed to the cell geometry (Figure 2a and inset; see Figures S2, S3, and S4, Supporting Information), as expected for thin graphenic sheets due to their relatively flexible $\mathrm{C}-\mathrm{C}$ bonds. ${ }^{5}$ Since the bacterial sections in Figure $2 \mathrm{a}$ are open, they dehydrate in TEM vacuum, producing wrinkles on PFGs and gaps between PFGs and bacterial cell walls. ${ }^{31}$

A live/dead test was conducted on the bacteria immediately before the wrapping process to confirm that the bacteria were alive (Figure $2 b$, inset) (see Supporting Information) with intact cell wall. ${ }^{32}$ This was important to make sure that the bacteria studied were completely hydrated. Owing to the impermeability of the PFG wraps, a live/dead test could not be conducted postwrapping.

For studying the effectiveness of graphenic wrapping under TEM conditions, the cells were immobilized on $100 \mathrm{~nm}$ thick silicon nitride $\left(\mathrm{Si}_{3} \mathrm{~N}_{4}\right)$ windows, followed by encasing the exposed face with PFG sheets. In a typical experiment, 20 

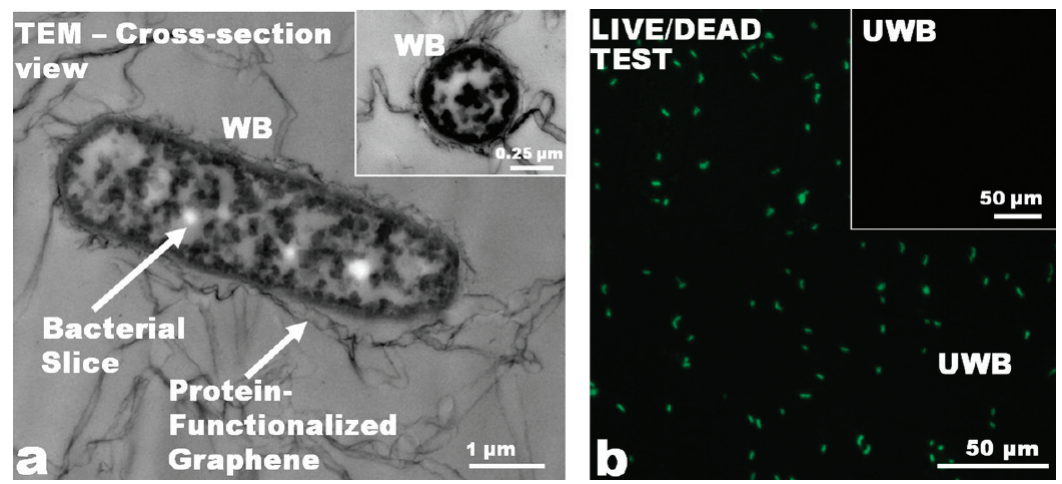

Figure 2. (a) TEM images of the $90 \mathrm{~nm}$ sections of wrapped bacteria showing the hermetic nature of the PFG wraps. Inset shows a lateral cross section of a wrapped bacterium. $90 \%$ of the bacteria were found to be completely wrapped. (b) Live/dead tests on the bacteria just before wrapping via Syto-9 (green fluorescence, live, shown by panel b) and PI (red fluorescence, dead: shown by inset) staining showing most of the bacteria to be alive.

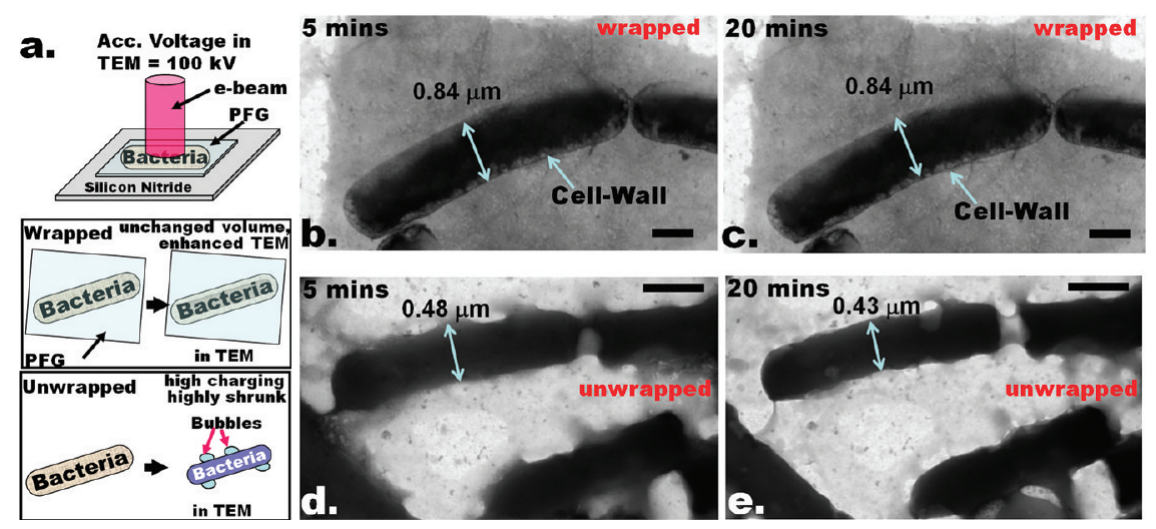

Figure 3. (a) PFG wrapping of bacteria prohibits shrinking under TEM. (b, c) Representative TEM images of wrapped bacterium (WB on $100 \mathrm{~nm}$ thick $\mathrm{Si}_{3} \mathrm{~N}_{4}$ windows) exhibits no shrinkage from the original size after 5 and 20 min exposure. (d, e) Representative unwrapped bacteria (UWB) exhibit $\sim 75 \%$ shrinkage after only 5 min under TEM vacuum (minimum time to obtain micrograph). Extensive bubbling is also observed during imaging, attributed to boiling of the volatile component of the cell's intracellular region (see videos in Supporting Information). Scale bar $=500 \mathrm{~nm}$. Note that under the same conditions, the cell wall of the wrapped bacteria is clearly discernible. This is attributed to significantly reduced charge accumulation due to the conductive PFGs ( $\pi$ electrons are highly conductive).

wrapped or unwrapped bacteria (from a fresh culture), immobilized on the $\mathrm{Si}_{3} \mathrm{~N}_{4}$ window, were analyzed. It is important to note that even in the same culture, different bacterium are at different stages of the cell cycle resulting in slight variations in their spatial dimensions. ${ }^{32}$ The average dimensions of the native bacteria were first recorded via an optical microscope at atmospheric pressure. The TEM micrographs were subsequently obtained after 5 (minimum time to start collecting images after stage insertion and microscope focus/contrast optimization), 15, and $20 \mathrm{~min}$ with the following conditions: vacuum of $10^{-5}$ Torr, incident current density of $150 \mathrm{~A} / \mathrm{cm}^{2}, 100 \mathrm{kV}$ electron beam, 1 pA probe current, $13500 \times$ magnification, spot size $\sim 1.5 \mu \mathrm{m}$ illumination diameter (CM100 spot size \# 6). For study of the electron-beam-induced damage, the side of the window with the bacteria was oriented toward the electron beam with the electron beam on at all times, while for exclusive vacuum study, the samples were oriented such that the electrons hit the $\mathrm{Si}_{3} \mathrm{~N}_{4}$ layer before the bacteria, with the only electron flux exposure during image acquisition ( $30 \mathrm{~s}$ for the $1024 \times 1024$ pixels). Under TEM, the samples were examined for (a) ability to resolve the cell wall, (b) retention of the bacterial dimensions, and (c) permeability (under vacuum and vacuum plus electron bombardment).

The TEM micrographs of the PFG wrapped bacteria show that the bacterial cell wall and intracellular structure are clearly discernible. This is attributed to (a) the high density of conductive $\pi$ electrons in the PFG sheets, which reduces the localized charge buildup, ${ }^{22,33}$ and (b) the high thermal conduction of the PFG sheet, which dissipates the heat generated from electron bombardment. ${ }^{9,22,33}$ In contrast, the TEM micrographs of the unwrapped bacteria under the same conditions exhibited charging, as evident from the dark images of the bacteria. Further, the cell wall and the other intracellular structure of the bacteria are unrecognizable.

Next, we analyzed unwrapped and wrapped cells for their size consistency under TEM. As mentioned above, the first TEM micrograph for each sample was obtained after the sample had resided in the TEM chamber for 5 min under a vacuum of $10^{-5}$ Torr; thus it depicts only the effect of vacuum on the bacteria. For the unwrapped bacteria, the initial average areal size (20 cells) at atmospheric pressure was $2.73 \pm 0.96 \mu \mathrm{m}^{2}(3.23 \pm 0.59$ $\left.\times 0.85 \pm 0.15 \mu \mathrm{m}^{2}\right)$, which reduced to $1.32 \pm 0.02 \mu \mathrm{m}^{2}(2.87 \pm$ $\left.0.23 \times 0.46 \pm 0.06 \mu \mathrm{m}^{2}\right)$ and $1.24 \pm 0.003 \mu \mathrm{m}^{2}(2.76 \pm 0.19 \times$ $0.45 \pm 0.06 \mu \mathrm{m}^{2}$ ) after $\sim 5$ and $20 \mathrm{~min}$ of exposure to vacuum and continuous electron beam exposure (Figure 3 and Table S1 in the Supporting Information). The observed $\sim 76 \%$ reduction in volume is attributed to the efflux of the internal hygroscopic intracellular molecules due to exposure to the high vacuum in the TEM column $\left(\sim 10^{-5}\right.$ Torr $)$ and due to the electron beam 

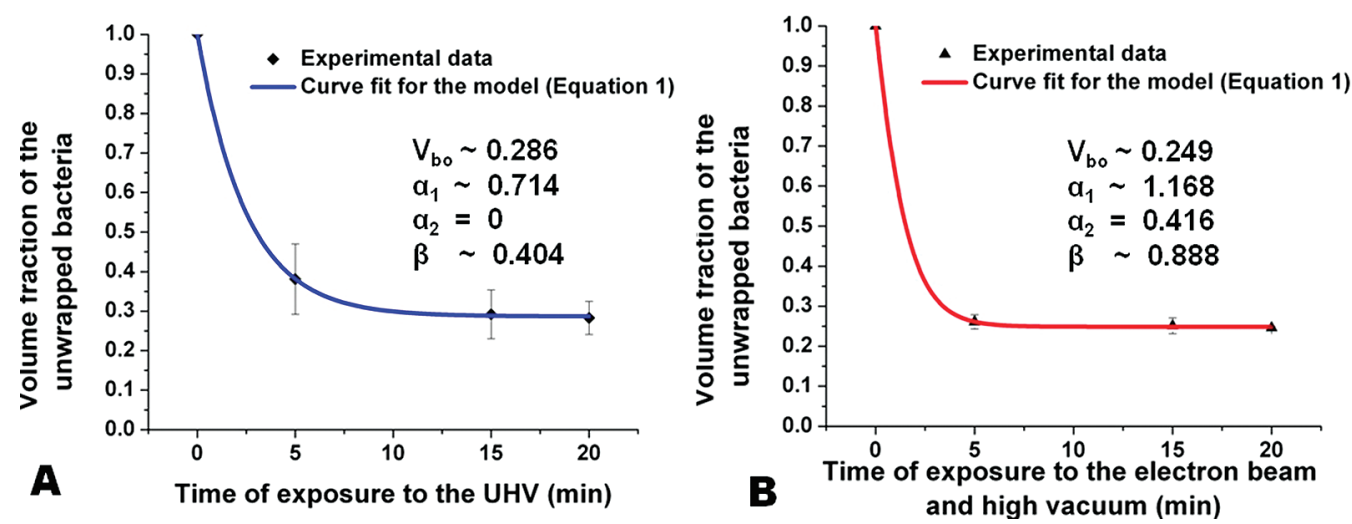

Figure 4. Efflux of intracellular matter in partially wrapped/unwrapped bacteria. (a, b) Curve fit of the model described in eq 1 with the normalized volume fraction shrinkage for the unwrapped bacteria exposed to vacuum and to vacuum plus electron beam. The error bars are the standard deviation of 20 measurements.

induced damage of the cell wall. Further, as shown in supplementary videos SV3 and SV4 (Supporting Information), the unwrapped bacteria also produced bubbles on the cell wall, attributed to the "boiling" of the intracellular volatile components under high vacuum. ${ }^{31,33}$ The high bubble formation in the unwrapped bacteria along with the charge buildup mentioned above indicate that the bacterial cell wall was being damaged significantly more than for the wrapped bacteria. ${ }^{2}$ In stark contrast, no change in the area $\left(2.897 \pm 0.565 \mu \mathrm{m}^{2}(3.438 \pm 0.028 \times\right.$ $\left.0.843 \pm 0.159 \mu \mathrm{m}^{2}\right)$ ) or structure was observed for fully wrapped bacteria after $\sim 20 \mathrm{~min}$ of vacuum and electron beam exposure (Figure 3; see supporting video SV1 and Tablex S1 and S2, Supporting Information). The least count of our measurements was $10 \mathrm{~nm}$ (for FEI-CM100 TEM). After $\sim 20 \mathrm{~min}$ of vacuum/ electron-beam exposure, the unwrapped bacteria were expected to be dry. This was vindicated by the experimentally observed volume percentage of volatile matter in bacteria of $\sim 76 \%$ (assuming isotropic cross-sectional shrinkage), consistent with literature. ${ }^{32,34}$ Further assuming that the majority of volatile matter is water, the water retention achieved by PFG wrapping was $\sim 350 \%$ volume (dry basis). This retention of intracellular volatile matter by the ultrathin graphenic wrapping is attributed to (a) the high impermeability of the graphenic lattice, where the high $\pi$-electron density on the carbon rings repels molecules and does not allow even helium to pass through ${ }^{5}$ and (b) high yield strength of graphene, where the lattice atoms are covalently linked in honeycomb structure, bestowing it with high stiffness. ${ }^{5,6,23}$ Expectedly, for the partially wrapped bacteria we observed 5-10\% volume shrinkage (assuming the bacterial shrinkage to be radially isotropic), which was attributed to the out-diffusion through unwrapped regions between the overlapping sheets and through the vacancy defects on GO from the harsh acid treatment (see supporting video SV5 and Figure S5 in the Supporting Information).

The mass-transfer (MT) resistance for release of volatile intracellular components from the bacteria is inversely proportional to the Darcian permeability, $k$, of the bacterial membrane. ${ }^{35}$ Further, the electron-flux-induced damage of cell wall is expected to open more MT channels, thus increasing the effective permeability. A simple bacterial-shrinkage model was constructed

$$
\mathrm{MT} \propto \frac{k}{R} C_{\mathrm{w}}+\frac{A I}{\lambda} C_{\mathrm{w}} C_{\mathrm{T}}
$$

where $k$ is the Darcian permeability, $A$ is the electron-beam damage factor, $C_{\mathrm{w}}$ and $C_{\mathrm{T}}$ are the water and total concentration in a bacterium, $R$ is the radius of the bacterium, $I$ is the electron beam current, and $\lambda$ is the latent heat of vaporization. This gives

$$
V=\frac{V_{\mathrm{B} 0}+\alpha_{1} \exp (-\beta t)}{1+\alpha_{2} \exp (-\beta t)}
$$

where $V_{\mathrm{B} 0}$ is the volume fraction of completely dehydrated bacteria, $\alpha_{1}$ and $\beta$ are constants inversely proportional to membrane permeability, and $\alpha_{2}$ is a constant proportional to the additional permeability caused by electron-beam-induced membrane damage. Additionally, the initial rate of volume shrinkage

$$
\left.\frac{\mathrm{d} V}{\mathrm{~d} t}\right|_{t=0}=\left(V_{\mathrm{B} 0}+\alpha_{1}\right) \frac{\alpha_{2} \beta}{\left(1+\alpha_{2}\right)^{2}}-\frac{\alpha_{1} \beta}{\left(1+\alpha_{2}\right)}=P^{\mathrm{D}}
$$

gives combined permeability $\left(P^{\mathrm{D}}\right.$, Darcian and electron-beam induced permeability).

For unwrapped bacteria, the values of $V_{\mathrm{B} 0}, \alpha_{1}, \alpha_{2}$, and $\beta$ were found to be $0.29,0.71,0$, and $0.40 \mathrm{~min}^{-1}$ for the effects of vacuum. Further, the Darcian permeability (with $\alpha_{2}=0$ ) is calculated to be $7.6 \times 10^{-9} \mathrm{~m} / \mathrm{s}$ (consistent with literature, ${ }^{36}$ see Supporting Information for details). For the combined effect of the electron beam and vacuum, the values of $V_{\mathrm{B} 0}, \alpha_{1}, \alpha_{2}$, and $\beta$ were found to be $0.25,1.17,0.42$, and $0.89 \mathrm{~min}^{-1}$ (Figure 4, see Supporting Information). The combined permeability, $P^{\mathrm{D}} \sim$ $20.5 \times 10^{-9} \mathrm{~m} / \mathrm{s}$, was an order of magnitude higher than that for the only vacuum exposure, reaffirming the adverse effects of the electron beam. It is important to note that the curve fits shown in Figure 4 are an extrapolation of the three data points, which were used to measure the Darcian permeability. The wrapped bacteria exhibited no discernible volume change; therefore the permeability was practically zero. Theoretically, if a monolayer graphene sheet wraps a bacterium under $10^{-5}$ Torr pressure, it experiences $\sim 1$ atm pressure difference. Under this pressure difference, graphene $(\text { strain }=0.26 \%)^{5}$ will stretch by $\sim 4.38 \mathrm{~nm}$. With an elastic modulus of $\sim 400 \mathrm{~N} / \mathrm{m}$, the average lateral force on graphene will then be $1.752 \mu \mathrm{N}(=E \times \Delta x)$ and the net force across the membrane of a bacterium would be $0.46 \mu \mathrm{N}(=\Delta P \times$ $\left.A_{\text {bacteria }}\right)$. In the current study, the graphenic multilayers with or without defects are expected to exhibit a higher resistance to stretching than graphene monolayers. ${ }^{21}$ This extrapolation from 
Bunch et $\mathrm{al}^{5}$ is consistent with the observed retention of the original volume of the PFG-encased bacteria under TEM (Figure 3). This further indicates that there was no slippage of the sheets either from the substrate or between different layers in the time scale of our measurements. It is pertinent to note that the electron transparency of graphene can cause electron-bombardment-induced hydrolysis of bacterial water to produce hydrogen (see Supporting Information). ${ }^{33}$ However, further studies are required to evaluate the effect of hydrolysis. Futuristically, the graphene-encased systems could benefit from covalently sealing (bonding) the overlapped graphene sheets to reduce intralayer diffusion (from between sheets).

In conclusion, we have demonstrated that bacteria can be encased within graphenic chamber by wrapping them with PFG. The unique structural, chemical, and electrical properties of graphene can then be applied to significantly improve their TEM imaging as compared to imaging native unwrapped cells: for example, (a) "wet" state TEM imaging of cells can be achieved; (b) bacteria can be imaged with original, uncontracted dimensions; (c) the bacterial volatile content can be retained; (d) internal bacterial structures, such as the cell wall, can be clearly distinguished in comparison to unwrapped bacteria under similar TEM conditions; (e) electrostatic charge buildup can be significantly reduced. Under similar TEM imaging, the unwrapped bacteria shrank by $76 \%$ and exhibited significant charge buildup. Postwrapping, the Darcian permeability of bacteria reduced from $7.6-20 \mathrm{~nm} / \mathrm{s}$ to practically zero. The specific graphenic properties which enable the above results include high impermeability, electron transparency, high conductivity, structural strength, and conforming flexibility. We envision that encasing wet samples within graphenic chambers will potentially enable real-time imaging of fluid dynamics, liquid suspensions of nanoparticles, proteins, and live cell's biochemical activity.

\section{ASSOCIATED CONTENT}

S Supporting Information. Description of methods and instruments used, TEM, FESEM, and confocal microscope images, TEM beam damage discussion, models, permebility calcualtions, and videos. This material is available free of charge via the Internet at http://pubs.acs.org.

\section{AUTHOR INFORMATION}

\section{Corresponding Author}

*E-mail: vberry@ksu.edu.

\section{ACKNOWLEDGMENT}

V.B. is grateful for the financial support from NSF (CMMI0939523), Terry C. Johnson Center for Basic Cancer Research, and KSU start-up. V.B. thanks Dr. Praveen Vadlani (KSU) for providing the Bacillus subtilis samples and Dr. David Moore (KU) for help with TEM and FESEM.

\section{REFERENCES}

(1) Williams, D. B.; Carter, C. B. Transmission electron microscopy; Springer: Berlin, 2009.

(2) Cellular Electron Microscopy; Mcintosh, J. R., Ed.; Methods in Cell Biology, Vol. 79; Academic Press: Boston, MA, 2007.

(3) Ayache, J.; Beaunier, L.; Boumendil, J.; Ehret, G.; Laub, D. Sample preparation handbook for transmission electron microscopy: techniques; Springer: Berlin, 2010.
(4) Geim, A. K.; Novoselov, K. S. The rise of graphene. Nat. Mater. 2007, 6, 183-191.

(5) Bunch, J. S.; Verbridge, S. S.; Alden, J. S.; van der Zande, A. M.; Parpia, J. M.; Craighead, H. G.; McEuen, P. L. Impermeable atomic membranes from graphene sheets. Nano Lett. 2008, 8, 2458-2462.

(6) Gomez-Navarro, C.; Burghard, M.; Kern, K. Elastic properties of chemically derived single graphene sheets. Nano Lett. 2008, 8, 20452049.

(7) Tsoukleri, G.; Parthenios, J.; Papagelis, K.; Jalil, R.; Ferrari, A. C.; Geim, A. K.; et al. Subjecting a Graphene Monolayer to Tension and Compression. Small 2009, 5, 2397-2402.

(8) Castro Neto, A. H.; Guinea, F.; Peres, N. M. R.; Novoselov, K. S.; Geim, A. K. The electronic properties of graphene. Rev. Mod. Phys. 2009, $81,109-162$.

(9) Balandin, A. A.; Ghosh, S.; Bao, W. Z.; Calizo, I.; Teweldebrhan, D.; Miao, F.; Lau, C. N. Superior thermal conductivity of single-layer graphene. Nano Lett. 2008, 8, 902-907.

(10) Parsons, D. F.; Matricardi, V. R.; Mortez, R. C.; Turner, J. N. Electron microscopy and diffraction of wet unstained and unfixed biological objects. Adv. Biol. Med. Phys. 1974, 15, 161-270.

(11) Hui, S. W.; Parsons, D. F. Electron-diffraction of wet biological membranes. Science 1974, 184, 77-78.

(12) Parsons, D. F. Structure of wet specimens in electron-microscopy. Science 1974, 186, 407-414.

(13) Gai, P. L. Development of wet environmental TEM (WetETEM) for in situ studies of liquid-catalyst reactions on the nanoscale. Microsc. Microanal. 2002, 8, 21-28.

(14) Heide, H. G. Electron microscopic observation of specimens under controlled gas pressure. J. Cell Biol. 1962, 13, 147-152.

(15) Williamson, M. J.; Tromp, R. M.; Vereecken, P. M.; Hull, R.; Ross, F. M. Dynamic microscopy of nanoscale cluster growth at the solid-liquid interface. Nat. Mater. 2003, 2, 532-536.

(16) Daulton, T. L.; Little, B. J.; Lowe, K.; Jones-Meehan, J. In situ environmental cell-transmission electron microscopy study of microbial reduction of chromium(VI) using electron energy loss spectroscopy. Microsc. Microanal. 2001, 7, 470-485.

(17) Liu, K. L.; Wu, C. C.; Huang, Y. J.; Peng, H. L.; Chang, H. Y.; Chang, P.; et al. Novel microchip for in situ TEM imaging of living organisms and bio-reactions in aqueous conditions. Lab Chip 2008, 8, 1915-1921.

(18) de Jonge, N.; Poirier-Demers, N.; Demers, H.; Peckys, D. B.; Drouin, D. Nanometer-resolution electron microscopy through micrometers-thick water layers. Ultramicroscopy 2010, 110, 1114-1119.

(19) Peckys, D. B.; Veith, G. M.; Joy, D. C.; de Jonge, N. Nanoscale Imaging of Whole Cells Using a Liquid Enclosure and a Scanning Transmission Electron Microscope. PLoS ONE 2009, DOI 10.1341/ journal.pone.0008214.

(20) de Jonge, N.; Peckys, D. B.; Kremers, G. J.; Piston, D. W. Electron microscopy of whole cells in liquid with nanometer resolution. Proc. Natl. Acad. Sci. U.S.A. 2009, 106, 2159-2164.

(21) Leenaerts, O.; Partoens, B.; Peeters, F. M. Graphene: A perfect nanoballoon. Appl. Phys. Lett. 2008, 93, No. 193107.

(22) Wilson, N. R.; Pandey, P. A.; Beanland, R.; Young, R. J.; Kinloch, I. A.; Gong, L.; Liu, Z.; Suenaga, K.; Rourke, J. P.; York, S. J.; Sloan, J. Graphene Oxide: Structural Analysis and Application as a Highly Transparent Support for Electron Microscopy. ACS Nano 2009, $3,2547-2556$.

(23) Lee, C.; Wei, X. D.; Kysar, J. W.; Hone, J. Measurement of the elastic properties and intrinsic strength of monolayer graphene. Science 2008, 321, 385-388.

(24) Beveridge, T. J.; Murray, R. G. E. How Thick is the BacillusSubtilis Cell-Wall. Curr. Microbiol. 1979, 2, 1-4.

(25) Mohanty, N.; Berry, V. Graphene-Based Single-Bacterium Resolution Biodevice and DNA Transistor: Interfacing Graphene Derivatives with Nanoscale and Microscale Biocomponents. Nano Lett. 2008, 8, 4469-4476.

(26) Hummers, W. S.; Offeman, R. E. Preparation of Graphitic Oxide. J. Am. Chem. Soc. 1958, 80, 1339-1339. 
(27) Archibal, A. R.; Coapes, H. E. The Interaction of ConcanavalinA with Teichoic Acids and Bacterial Walls. Biochem. J. 1971, 123, 665667.

(28) Kim, J.; Cote, L. J.; Kim, F.; Huang, J. X. Visualizing Graphene Based Sheets by Fluorescence Quenching Microscopy. J. Am. Chem. Soc. 2010, 132, 260-267.

(29) Prausnitz, J. M.; Lichtenthaler, R. N.; Azevedo, E. G. Molecular Thermodynamics of Fluid-Phase Equilibria; Prentice-Hall: Englewood Cliffs, NJ, 1998.

(30) Bouckaert, J.; Loris, R.; Poortmans, F.; Wyns, L. Crystallographic structure of metal-free concanavalin $\mathrm{A}$ at 2.5 angstrom resolution. Proteins: Struct., Funct., Bioinf. 1995, 23, 510-524.

(31) Egerton, R. F.; Wang, F.; Crozier, P. A. Beam-induced damage to thin specimens in an intense electron probe. Microsc. Microanal. 2006, $12,65-71$.

(32) Lodish, H.; Berk, A.; Zipursky, L.; Matsudaira, P.; Baltimore, D.; Darnell, J. Molecular cell biology; W. H. Freeman: New York, 1999.

(33) Egerton, R. F.; Li, P.; Malac, M. Radiation damage in the TEM and SEM. Micron 2004, 35, 399-409.

(34) Bratbak, G.; Dundas, I. Bacterial dry-matter content and biomass estimations. Appl. Environ. Microbiol. 1984, 48, 755-757.

(35) Incropera, F. P. Fundamentals of heat and mass transfer; Wiley: New York, 2006.

(36) deMaranon, I. M.; Gervais, P.; Molin, P. Determination of cells' water membrane permeability: Unexpected high osmotic permeability of Saccharomyces cerevisiae. Biotechnol. Bioeng. 1997, 56, 62-70. 